\title{
EL OBJETO DE ESTUDIO EN CIENCIAS SOCIALES: ENTRE LA PREGUNTA Y LA HIPÓTESIS
}

\section{THE OBJECT OF STUDY IN SOCIAL SCIENCES: BETWEEN QUESTION AND HYPOTHESIS}

Mg. César Tello (cesargeronimotello@yahoo.com.ar) Escuela de Humanidades, Universidad Nacional de San Martín (Buenos Aires, Argentina)

\begin{abstract}
This paper presents and analyses by way of an open debate certain issues inherent to the choice of researchers when defining the epistemological axis of his research: question or hypothesis. It has been long considered that these components must be present simultaneously in a research project, when in fact belong to different epistemological axes. Each one is different and powerful enough to start some research. But the simultaneous presence of both axes removes epistemological strength and consistency to a research project because the different rationale of each of these axes.
\end{abstract}

Keywords: research project, object of study, axis epistemology, question, hypothesis

\section{Resumen}

En este trabajo, se presentan y analizan, a modo de debate ciertas problemáticas inherentes a la elección de los investigadores al momento de definir el eje epistemológico de su investigación: pregunta o hipótesis. Durante mucho tiempo se ha considerado que estos componentes deben estar presentes simultáneamente en un proyecto de investigación, cuando en realidad pertenecen a lógicas diversas. Aquí planteamos la necesidad de reflexionar sobre ejes epistemológicos distintos y a la vez potentes para iniciar una investigación. Pero la co-presencia de ambos ejes en la investigación le quita solidez y consistencia epistemológica a un proyecto de investigación, dado que la racionalidad subyacente con que se construye cada uno de estos ejes difiere entre sí.

Palabras clave: proyecto de investigación, objeto de estudio, eje epistemológico, pregunta, hipótesis.

\section{Introducción}

Este artículo tiene por objeto presentar y analizar algunas dificultades que se encuentran al momento de optar por el eje epistemológico (EE) de un proyecto de investigación, esto es: decidir si será una pregunta o una hipótesis la que se constituirá en el componente central del objeto de estudio. El investigador, en general, utiliza uno u otro EE y, en algunos casos ambos de modo simultáneo al momento de diseñar su proyecto, sin considerar los diversos caminos que tomará su indagación si opta por la pregunta o la hipótesis. Entendiendo que el objeto de estudio se constituye en la columna vertebral del proyecto de 
investigación y, esa vertebralidad posee un EE que la define: la pregunta o la hipótesis. Es necesario señalar que el EE del objeto de estudio atravesará todos los componentes del proyecto de investigación. En este sentido no es menor la reflexión del investigador acerca de la decisión sobre la opción entre pregunta o hipótesis.

Aquí no hablaremos de "mejores o peores" ejes de un proyecto de investigación, cuestión que es común encontrar en algunas reflexiones epistemológicas, particularmente cuando se refieren a las metodologías cualitativas y cuantitativas, asociando erróneamente las primeras al EE de investigación "pregunta" y las segundas a la "hipótesis". Siguiendo en esta línea de análisis es que consideramos que no es apropiado denominar a las metodologías cualitativas y cuantitativas como paradigmas. Con esto decimos que los paradigmas, desde las múltiples acepciones con que se la quiera utilizar (Khun, Saussure, Fleck, entre otros) están vinculados a enfoques teóricos que pueden devenir en uno u otro modo de construcción metodológica, como desarrollamos en el primer apartado. Considerando que del enfoque teórico seleccionado por el investigador se desprende de lo que hemos denominado posición epistémica del investigador. Entendiendo así que el paradigma asumido en el proyecto de investigación no se define por las metodologías cualitativas o cuantitativas de investigación. Aquí se da lo que denomino la falacia epistemológica de las metodologías y la posición epistémica. Esta falacia se produce cuando la metodología es tomada como paradigma en tanto se mencionan las estrategias metodológicas como "paradigma cualitativo" o "paradigma cuantitativo" cuando en realidad no lo son. Es decir, la falacia se produce en tanto desde lo metodológico se intenta establecer un paradigma $y$, el paradigma con que se desarrolla un proyecto de investigación se encuentra en la posición epistémica del investigador, empleando uno u otro modo metodológico.

Esto no significa que no exista relación entre metodología y enfoque teórico y posición epistémica del investigador, pero no son inherentes, mecánicas o causales. Con esto quiero decir que una investigación en ciencias sociales puede asumir un paradigma positivista y utilizar algunos componentes de la metodología cualitativa para el despliegue metodológico.

El análisis que desarrollamos se extiende en torno al énfasis de un proyecto de investigación, sea en una lógica del descubrimiento o una lógica de la verificación. Es aquí donde observamos una gran dificultad en términos metodológicos de los proyectos de investigación que afectan la consistencia y coherencia epistemológica: cuando se confunde opción por un enfoque teórico por parte del investigador asumiendo una posición epistémica con la metodología como paradigma. Esto se puede observar en investigadores jóvenes y en determinada bibliografía que vincula la lógica de la verificación con la metodología cuantitativa y la lógica de descubrimiento con la metodología cualitativa. De este modo presento las categorías claves de este trabajo:

a) El objeto de estudio como columna vertebral del proyecto de investigación.

b) La pregunta o hipótesis como eje epistemológico del objeto de estudio.

c) Las metodologías cualitativas y cuantitativas como estrategias para el abordaje, pero no como paradigmas. 
d) El enfoque teórico -como paradigma- del investigador y la posición epistémica que se desprende del enfoque. A modo de ejemplo podemos mencionar que el investigador asume el enfoque teórico del neomarxismo y la posición epistemológica del neo-marxismo latinoamericano.

\section{Eje inicial, objeto de estudio y etapas del proceso de investigación}

En primer lugar debemos considerar que uno u otro eje epistemológico inicial son válidos para desarrollar una investigación. Ahora bien, para poder enhebrar una lógica coherente y consistente en el desarrollo de una investigación en ciencias sociales, es necesario considerar en palabras de Andrade que: "la crítica no es al empleo de hipótesis ni mucho menos a la definición de preguntas de investigación en sí mismos, si no a la poca reflexión que un uso mecánico de tales procedimientos da lugar" (2007:265).

Una de las grandes dificultades que tiene la enseñanza de la metodología de investigación en ciencias sociales en los espacios académicos latinoamericanos tiene que ver con la transmisión técnico-organizativa de lo que es un diseño o proyecto de investigación (1), desarrollando a veces, de modo instrumental algunos conceptos y categorías que se constituirán en los componentes de un proyecto de investigación. La enseñanza universitaria en metodología de la investigación en ciencias sociales, tanto en el grado como el posgrado en Latinoamérica es fuertemente prescriptiva (Barriga y Henríquez 2003). Aquí es interesante pensar que dado el desarrollo y reflexiones de la epistemología en los últimos treinta años que provocó el pasaje de "el método científico" a "los métodos científicos" produjo por efecto sumativo nuevas categorías y componentes en un diseño de investigación generando confusión epistemológica en el uso de pregunta e hipótesis, observando que en algunos casos son utilizados casi como sinonimia y en otros como pasos sucesivos. Esto se puede observar en universidades de Latinoamérica y/o en agencias nacionales de investigación en cuyas planillas de aplicación para la presentación de proyectos de investigación se observan los componentes: "-Pregunta -Hipótesis..." continuando así con el los otros componentes de las planillas y formularios del proyecto de investigación. Sin dejar al investigador la posibilidad de seleccionar uno u otro EE.

Bolívar afirma que dentro del denominado giro hermenéutico producido en los años sesenta en las ciencias sociales, se produjo un pasaje importante "de la instancia positivista a una perspectiva interpretativa" (2002:3). En medio de este giro en ciencias sociales muchos investigadores se sintieron más cómodos y les resultaba más apropiado el EE de la pregunta para definir su objeto de estudio. Pero con el temor de "no perder" la denomina rigurosidad científica y ante el riesgo que su proyecto corría de no ser tomado dentro de los cánones de la rigurosidad a la que nos referíamos, seguían empleando la hipótesis, más aún: empleaban la hipótesis e introducían la pregunta. Es así como comienza a verse de modo simultáneo en las investigaciones la co-presencia de ejes epistemológicos.

Así, de modo cronológico en las décadas de 1960 y 1970 se produce la transmisión conceptual en los espacios de formación académica de este nuevo EE: la pregunta. Sin considerar el contexto en que fueron generadas y muchas veces tomadas desde el sentido común, extirpando, de algún modo, la perspectiva epistemológica de los ejes, componentes y etapas de una investigación: "la investigación es enseñada como el modo en que se hace investigación, no se revisan sus orígenes, se transmite como un acervo de herramientas y estrategias para hacer" (Andrade 2007:264). 
En general se puede observar en investigadores sin experiencia cierta confusión inicial en el proceso de construcción del proyecto de investigación, desde nuestra exploración de treinta y ocho proyectos de investigación analizados en Universidades Nacionales de Argentina, Colombia, México, Brasil y Uruguay y el contacto que tenemos con los propios alumnos en Seminarios de Elaboración de Proyectos de Tesis tanto del grado como de posgrado, observamos que luego de haber cursado los seminarios de metodología (2) previos al seminario de Taller de Tesis, optan de modo simultáneo y, sin mucha reflexión, por la pregunta e hipótesis como EE para comenzar a construir su objeto de estudio.

Pero estas dificultades no son sólo atribuibles a los investigadores que se inician, sino a cierta literatura muy utilizada en los ámbitos de enseñanza de metodología de investigación -como espacio curricular previo a la construcción del proyecto de investigación- que despliegan esta confusión, aquí presentamos algunos autores que explican los pasos de un proceso de investigación para comprender a que nos estamos refiriendo, y tomamos particularmente autores que se encuentran fácilmente en los programas de los seminarios de metodología en países latinoamericanos.

Cuadro I: Algunos autores que utilizan de modo simultáneo Pregunta e Hipótesis (Elaboración propia)

\begin{tabular}{|c|c|c|c|c|c|}
\hline Salkind (1999) & Bunge (1993) & $\begin{array}{c}\text { Sampieri et. al. } \\
\text { (2003) }\end{array}$ & Mendez (1994) & Borsotti (2008) & Samaja (2005) \\
\hline $\begin{array}{l}\text { 1. Formulación de } \\
\text { un Pregunta. } \\
\text { 2. Identificar } \\
\text { factores } \\
\text { importantes. } \\
\text { 3. Formulación de } \\
\text { hipótesis de } \\
\text { investigación. } \\
\text { 4. Recopilación de } \\
\text { la información. } \\
\text { 5. Probar la } \\
\text { Hipótesis. } \\
\text { 6. Trabajar con la } \\
\text { hipótesis. } \\
\text { 7. Reconsideración } \\
\text { de la teoría. } \\
\text { 8. Confirmación o } \\
\text { refutación. }\end{array}$ & $\begin{array}{l}\text { 1. Definición del } \\
\text { Pregunta. } \\
\text { 2. Hipótesis; como } \\
\text { el modo de pensar } \\
\text { o imaginar una } \\
\text { respuesta probable } \\
\text { al mismo } \\
\text { 3. Hipótesis (o } \\
\text { subhipótesis) } \\
\text { empíricas. } \\
\text { 4. Diseño de la } \\
\text { verificación de las } \\
\text { hipótesis o del } \\
\text { procedimiento } \\
\text { concreto a seguir } \\
\text { en su prueba. } \\
\text { 5. Puesta a prueba } \\
\text { o contraste con la } \\
\text { realidad de la } \\
\text { hipótesis a través } \\
\text { de sus } \\
\text { consecuencias o } \\
\text { mediante } \\
\text { subhipótesis } \\
\text { empíricas. } \\
6 . \text { Establecimiento } \\
\text { de las conclusiones } \\
\text { resultado de la } \\
\text { investigación y } \\
\text { 7. Extensión las } \\
\text { conclusiones o } \\
\text { generalizar los } \\
\text { resultados. }\end{array}$ & $\begin{array}{l}\text { 1. Concebir la idea } \\
\text { 2. Planteamiento } \\
\text { de la Pregunta } \\
\text { 3. Elaboración del } \\
\text { marco teórico } \\
\text { 4. Establecimiento } \\
\text { de la hipótesis } \\
\text { 5. Selección del } \\
\text { diseño apropiado } \\
\text { 6. Selección de la } \\
\text { muestra } \\
\text { 7. Recolección de } \\
\text { datos } \\
\text { 8. Análisis de datos } \\
\text { 9. Presentación de } \\
\text { los resultados }\end{array}$ & $\begin{array}{l}\text { 1. Definición del } \\
\text { tema investigación } \\
\text { 2. Planteamiento } \\
\text { del Pregunta } \\
\text { 3. Formulación y } \\
\text { sistematización } \\
\text { del Pregunta de } \\
\text { investigación } \\
\text { 4. Objetivos de la } \\
\text { investigación } \\
\text { 5. Justificación } \\
\text { 6. Marco de } \\
\text { referencia } \\
\text { 7. Hipótesis } \\
\text { 8. Aspectos } \\
\text { metodológicos } \\
\text { 9. Bibliografía } \\
\text { 10. Cronograma. }\end{array}$ & $\begin{array}{l}\text { 1. Situación } \\
\text { problemática } \\
\text { 2. Construcción } \\
\text { del objeto } \\
\text { (conocimiento } \\
\text { disponible) } \\
\text { 3. Formulación de } \\
\text { las preguntas } \\
\text { 4. Objetivos de la } \\
\text { Investigación } \\
\text { 5. Hipótesis } \\
\text { 6. Obtención de } \\
\text { información } \\
\text { 7. Procesamiento } \\
\text { y sistematización } \\
\text { de la información } \\
\text { 8. Cronograma } \\
\text { 9. Presupuesto } \\
\text { 10. Bibliografía }\end{array}$ & $\begin{array}{l}\text { 1. Planteamientos } \\
\text { Preliminares a) } \\
\text { Preguntas; b) } \\
\text { Hipótesis; c) } \\
\text { fundamentos; d) } \\
\text { Propósitos. } \\
\text { 2. Formulaciones de } \\
\text { Preguntas; a) } \\
\text { Hipótesis; b) } \\
\text { fundamentos; c) } \\
\text { Propósitos. } \\
\text { 3. Diseño del Objeto } \\
\text { de Estudio. a) } \\
\text { unidades de análisis; } \\
\text { b) variables; c) } \\
\text { fuentes; d) } \\
\text { definiciones } \\
\text { operacionales. } \\
\text { 4. Diseño de los } \\
\text { Procedimientos a) } \\
\text { Muestra; b) Plan de } \\
\text { Análisis; c) plan en los } \\
\text { contextos; d) } \\
\text { instrumentos. } \\
\text { 5. Recolección y } \\
\text { Procesamiento de } \\
\text { Datos. a) Pilotajes; b) } \\
\text { Recolección; c) } \\
\text { Procesamiento de los } \\
\text { Datos; d) } \\
\text { Presentación. } \\
\text { 6. De Tratamiento y } \\
\text { Análisis de los Datos } \\
\text { Compactación de las } \\
\text { matrices; a) Pruebas; } \\
\text { b) Interpretación; c) }\end{array}$ \\
\hline
\end{tabular}




\begin{tabular}{|l|l|l|l|l|}
\hline & & & & $\begin{array}{l}\text { Conclusiones. } \\
\text { 7. De Elaboración de } \\
\text { Informes } \\
\text { a) Balance de } \\
\text { actividades; b) } \\
\text { ordenamiento de } \\
\text { resultados; c) } \\
\text { evaluación; d) } \\
\text { redacción de } \\
\text { informes } \\
\text { 8. De Exposición } \\
\text { Sistemática } \\
\text { a) Destinatarios; b) } \\
\text { Estructura retórica; c) } \\
\text { Desarrollo de } \\
\text { argumentaciones; d) } \\
\text { Correcciones finales. }\end{array}$ \\
& & &
\end{tabular}

En este cuadro se observa como, por ejemplo, en el caso de Bunge en el punto 2 comienza la dificultad técnico-epistemológica dado que el autor indica "pensar o imaginar una respuesta probable al mismo" (1993:35) y como sinónimo afirma "o hipótesis" (1993:35) en esta misma línea tomamos otro autor, no incluido en el cuadro, Ander-Egg, autor muy difundido en la investigación en educación afirma que "la hipótesis es una tentativa de explicación mediante una suposición o conjetura verosímil” (1995:14).

En el caso de Borsotti se puede observar que explica la hipótesis como "una conjetura provisoria" (2008:77), utilizando el lenguaje cotidiano, que consideramos es el que más confusión trae al momento de los debates metodológicos sobre estas cuestiones: "a veces se trata de hipótesis de trabajo, que toman la forma de supuestos. Cuando la pregunta es: ¿cuál es la relación entre ' $r$ ' y ' $z$ '? el supuesto que se toma como hipótesis es que habría alguna relación entre esos elementos" (Borsotti 2008:77, las negritas son mías).

Para Hernández Sampieri, las hipótesis son las guías de la investigación, entendiendo que "la hipótesis indica lo que estamos buscando, y se define como una explicación tentativa del fenómeno estudiado" (2003:140).

Esta confusión conceptual siguiendo las definiciones de los autores se resolvería si tomaran la hipótesis como tal y, se referirían a las explicaciones que dan sobre ellas del modo apropiado, por ejemplo: supuestos del investigador, anticipación de sentido (Sirvent 1999), lo que en la perspectiva anglosajona se puede encontrar como ideas orientadoras que ayudarían a guiar la investigación "sensitizing concepts" que permite según Herbert Blumer "una sensación general de referencia" (1954:7) o el desarrollo realizado por Bowen en Grounded Theory and Sensitizing Concepts (2006), entre otros. El planteamiento latentes o juicios predictivos (Zemelman 1992), intuición (Orozco 2005), ideas previas del investigador (Sabino 1996) y presupuesto teórico (Wainerman y Sautu 2001).

En ningún caso negamos los supuestos con los que un investigador comienza a desarrollar su proyecto, de hecho selecciona un tema y no otro, se hace algunas preguntas y no otras, etc., pero esto que le sucede al investigador debe ser llamado de modo apropiado para no generar confusiones terminológicas que pueden dar como resultado una inconsistencia en el proyecto de investigación. Y esto que le sucede al investigador supuestos- sucede tanto cuando define una hipótesis como una pregunta. El investigador que se plantea una pregunta o una hipótesis "no lo hace en el vacío, como si no tuviese la menor idea del mismo, sino que siempre parte de algunas ideas o informaciones previas, de algunos referentes teóricos y conceptuales, por más que éstos no tengan todavía un carácter preciso y sistemático" (Sabino 1996:51).

En este sentido Wainerman al referirse a los errores comunes en la investigación social explica que uno muy común es: "la confusión entre hipótesis como conjetura a someter a prueba e hipótesis como presupuesto o 
punto de partida que orienta la investigación. Es harto común que los estudiantes confundan el concepto de presupuesto teórico con el de hipótesis" (Wainerman y Sautu 2001:29).

Daré un ejemplo de una alumna, cuando planteaba: "La presente investigación tiene la siguiente hipótesis: el apoyo tutorial hace importante contribuciones en los alumnos con problemas de disciplina en la escuela". Este era el supuesto de la alumna. Que la presencia de determinado actor generaba ciertos beneficios en alumnos con determinadas dificultades. Es decir, allí existía un supuesto. Desde una perspectiva metodológica no estaba construido como pregunta ni como hipótesis. De hecho no se había podido aún construir el EE del objeto de estudio: pregunta o hipótesis.

En fin, el problema de la conjunción errónea que se hace de la presentación de hipótesis y pregunta en un proyecto de investigación radica, precisamente, en su formulación y modo de construcción. Una, la lógica de la verificación: la hipótesis, y otra la lógica del descubrimiento: la pregunta.

Esto se puede observar claramente en el esquema de Bunge cuando habla de hipótesis o sub-hipótesis empíricas y así escinde el objeto de estudio que, para el autor sería el esquema teórico de la base empírica. Esta división, como dijimos, responde al modelo de verificación, es decir, esto se da cuando con una teoría se va a verificar la realidad. Desde nuestra perspectiva, el objeto de estudio es en sí mismo una amalgama de teoría y lo empírico. En este sentido Zemelman explica: "el proceso constructor del objeto consiste en reconstruir un campo articulado con base en una pregunta-eje que sirva de punto de partida del conocimiento. Corresponde al esfuerzo de pensar de acuerdo con los requerimientos de un campo de posibilidades, en forma que pueda llegarse a fijar el contenido teórico de una proposición, rompiendo con la fragmentación de la observación sobre la realidad, mediante las articulaciones posibles de la pregunta eje (...) [que] refleja el esfuerzo por penetrar en la realidad por medio de una lógica de inclusividad de niveles que operan mediante instrumentos conceptuales que cumplen la función de reflejar la articulación y su movimiento vertical longitudinal, coyuntural - período" (1992:196).

No debemos confundir, como se hace regularmente, la dimensión teórica del objeto de estudio con la hipótesis y la dimensión empírica del mismo con la pregunta. Dado que el objeto de estudio posee las dos dimensiones: teoría y lo empírico, imbricadas entre sí, sea tanto en una hipótesis como en una pregunta. Pero en ocasiones se puede observar que se incluye la hipótesis creyendo que esta se vincula con las cuestiones teóricas y la pregunta considerando que se relaciona con los aspectos empíricos. Escindiendo así nuevamente el objeto de estudio y aunando, como veremos más adelantes, EE distintos. Este es un error metodológico que suele cometerse al no conocer el rol de cada uno de los ejes de un proyecto de investigación.

\section{El eje de investigación: ¿pregunta o hipótesis?}

Como hemos indicado, en la construcción del objeto se define el modelo con el que se diseñará el proyecto de la investigación: de verificación o de descubrimiento. Ahora bien, por qué decimos que uno u otro componente (pregunta o hipótesis) pertenecen a distintos modelos, considerando, claro está, que ambos son componentes potentes para la investigación en ciencias sociales. Hacemos esta afirmación por el modo en que se construye cada uno de esos componentes en el diseño de investigación.

\section{Construcción de la hipótesis}

Las hipótesis sostienen afirmaciones que se tratarán de probar. Pueden definirse como explicaciones tentativas del fenómeno que se investiga formuladas a manera de proposiciones. Klimovsky afirma que "quien plantea una hipótesis supone que ella es verdadera" (2001:132). Estas proposiciones se establecen como relaciones entre dos o más variables y se apoyan en conocimientos organizados. No puede existir desconocimiento sobre la hipótesis planteada, porque se puede cometer un error en su construcción. En 
este sentido podemos encontrar una serie de sugerencias o recomendaciones al momento de construir la hipótesis. Haremos una breve síntesis tomando el desarrollo conceptual de varios autores (McMillan y Schumacher 2005, Kerlinger 1979, Krathwohl 1988, Buendía, Colas y Hernandez 2000, Mejía 2005, Goode y Hatt 1970, Bunge 1993).

\section{La Hipótesis:}

-Debe ser lógica, debe ser coherente en términos de una explicación razonable que resista un análisis crítico; no puede ser descabellada hasta el punto de ser absurda.

-Debe poseer nivel de generalidad. La explicación es de carácter general y trasciende a una explicación o conjetura de hechos singulares; la hipótesis debe abarcar a una categoría de fenómenos que tengan algún atributo en común. Por otra parte, no debe ser tan general que impida precisar los conceptos y operaciones que de ella se desprendan.

-Posee una referencia empírica. Sus afirmaciones guardan relación con el mundo de los fenómenos observables.

-Debe ser comprobable y verificable. Si la explicación no permite someterla a prueba mediante los procedimientos metodológicos, no tiene validez. La lógica científica afirma que lo que da valor a cierta hipótesis es permitir ser falseada, es decir que luego de ser puesta a prueba en reiteradas situaciones para rechazarla, logra salir adelante sin objeciones.

-Debe tener operacionalidad, es decir, que sus términos sean claros, sin ambigüedades a fin que se puedan establecer las relaciones entre las variables y sus indicadores que permitirán observar su comportamiento.

-Debe tener una referencia teórica. Es preciso que se inserte en un cuerpo de teoría en forma explícita, a fin de procurar incrementar el acervo científico. La ciencia es acumulativa y una hipótesis aislada no aporta nada.

Luego de construida la hipótesis se continúa con un modelo y lógica implícita e inherente por su propio modo de construcción. Con esto decimos que la investigación cuyo objeto de estudio se plantee en término de hipótesis tendrá las siguientes características, siguiendo el modelo hipotético de Ayala (2001:55):

-Observar la información empírica sin realizar interpretaciones.

-Buscar regularidades y relaciones en la información.

-Formular la información general que dé cuenta de las regularidades o relaciones observadas.

-Hacer otras observaciones para evaluar si la información se mantiene.

La práctica de usar hipótesis se deriva del empleo del "método científico" en la investigación social. Hay ventajas filosóficas en el uso de la contrastación estadística, siempre que los investigadores sean cuidadosos y conservadores en la formulación de sus conclusiones (Armstrong 1974).

Según Pajares (2007) las hipótesis pueden ser divididas en cuatro tipos

1. Nula Literaria. No hay diferencias en término de constructos teóricos. Por ejemplo, "No existe relación entre los servicios de apoyo institucional y la persistencia académica de las mujeres de edad no tradicional que asisten a la facultad". "No hay diferencia entre el rendimiento académico de los alumnos con autoregulación alta y baja". 
2. Nula Operacional. No hay diferencias en términos de las operaciones necesarias para contrastar las hipótesis. En el ejemplo anterior, "no existe relación entre el número de horas que las mujeres de edad no tradicional utilizan el centro de estudiantes y su permanencia en la facultad después del primer año". "No hay diferencias entre las medias de calificaciones obtenidas por los estudiantes del primer y el tercer cuartil de la distribución de las puntuaciones del inventario de autoregulación".

3. Alternativa Literaria. En este caso se presenta la hipótesis que se aceptará en caso de que la hipótesis nula sea rechazada, en términos de constructos teóricos. En otras palabras, es lo que se espera que muestren los resultados. Por ejemplo, "cuando más utilicen los servicios de apoyo las mujeres de edad no tradicional, mayor será su persistencia académica". "Los alumnos de autorregulación alta tendrán mejor desempeño académico que los alumnos de autorregulación baja".

4. Alternativa Operacional. Este tipo de hipótesis es semejante al anterior, sólo que especificando las operaciones de medición. Por ejemplo, "cuando más utilicen el centro de estudiantes las mujeres de edad no tradicional, más persistirán académicamente después de su primer año de universidad". "Los estudiantes pertenecientes al tercer cuartil de la distribución del inventario de autorregulación tendrán notas significativamente mayores que los alumnos del primer cuartil”.

En general, la hipótesis nula es utilizada si la teoría o la literatura no sugiere una relación hipotética entre las variables investigadas; la alternativa se reserva generalmente para situaciones en las que la teoría o la investigación sugiere relación o interdependencia.

\section{Construcción de la Pregunta}

La pregunta de investigación se caracteriza por contener "todas aquellas cuestiones relacionadas con razones y motivos de los desequilibrios, rupturas, contradicciones, insatisfacciones y conflictos en que se involucra el sujeto cognoscente" (Becerra 1994:19). Principalmente, y a diferencia de la hipótesis, no se tienen dos posibles respuestas (de afirmación o negación de la hipótesis). El investigador desconoce a priori las múltiples relaciones, conflictos, estados de situación, etc. que arrojará su investigación. Esto es "la pregunta de investigación es el desconocimiento de algún asunto de interés" (Becerra 1994:27). Una pregunta tiene en sí mismo el carácter de incertidumbre. Pero la incertidumbre es propia de la pregunta de investigación. Si no hay incertidumbre, no hay pregunta.

En este sentido una investigación planteada en términos de pregunta, no requiere mucho trabajo previo, más que el interés del investigador, la definición del objeto en términos de pregunta y el diseño metodológico, a diferencia de la investigación basada en la hipótesis que requiere de mucho estudio previo por parte del investigador (porque necesita conocer la situación que tendrá que refutar o aceptar). En muchas ocasiones el investigador asume que debe construir su objeto de estudio en torno al EE de la hipótesis, porque siempre se hizo así, sin embargo Andrade advierte a modo de reflexión: “¿por qué debo emplear hipótesis en la investigación?, ¿qué implicaciones trae consigo el empleo de hipótesis en lo que se refiere al recorte del campo de observación?, ¿es posible plantear una investigación de modo distinto a la forma tradicional?, ¿cuál y qué características tendría esa forma alternativa?” (2007:263).

Zemelman denomina la pregunta como problema-eje afirmando que: "conocer es especificar y especificar es delimitar las relaciones de articulación que posee el problema respecto de otros fenómenos de la realidad. Este conjunto de relaciones es el contexto especificador dentro del cual el problema-eje adquiere su significado (...) de tal modo que la transformación del problema implica un doble cuestionamiento: de la teoría contenida en la definición del mismo, pues se exige a ésta subordinarse a las exigencias del 
razonamiento; y de lo empírico-morfológico, ya que al abandonar la fijeza aparente en que se presenta, se exige a éste mostrarse en sus cambios posibles" (1987:60).

Aquí se puede observar una advertencia: el problema-eje, para Zemelman, posee dos dimensiones (teoría y lo empírico) que se conjugan para abandonar la supuesta inmovilidad del objeto. Oponiéndose a la idea de objeto de estudio como componente teórico del proyecto de investigación. Así el problema-eje de Zemelman es un razonamiento problematizador de la realidad, y siguiendo con esta argumentación analizar y estudiar la realidad no consiste "en la recolección de datos empíricos", sino en la construcción de los datos desde una mirada específica que tiene que ver con lo que he denominado posicionamiento epistémico del investigador, esto es, la mirada y posición teórica, política y ética.

También es bien interesante plantear que en algunas ocasiones una pregunta es planteada en términos de hipótesis y no en términos de problema, que es otro error corriente en la construcción de un proyecto de investigación. ¿Cuándo sucede esto? Cuando la pregunta se responde con un "SI", o con un "NO" o porque sí o porque no. Esto significa que se ha incorporado "la respuesta” a la pregunta. Por ejemplo: “¿Por qué los alumnos de autorregulación alta tiene mejor desempeño académico que los alumnos de autorregulación baja?" Claramente se observa la hipótesis mal formulada como pregunta, la hipótesis sería: los alumnos de autorregulación alta tiene mejor desempeño académico que los alumnos de autorregulación baja y, si se quiere trasladar esta situación investigativa a una pregunta debería ser: ¿qué relación existe entre la autorregulación de los alumnos y el desempeño académico?

\section{Contextos/Lógicas de descubrimiento y Verificación/Justificación}

Debemos considerar que la distinción entre "contexto de descubrimiento y contexto de justificación" fue desarrollada por H. Reinchebach en su obra "Experience and Prediction" de 1934 para diferenciar las etapas en que se llevan a cabo los descubrimientos en investigación y el modo en que se justifican o aceptan.

Es necesario considerar que ambos contextos, en su definición original, se encuentran presentes tanto en el proceso de construcción del proyecto de investigación como de la investigación en sí misma. Ahora bien, en los diversos debates epistemológicos la categoría contexto de descubrimiento y de verificación pasó a desarrollarse o conceptualizarse como sinonimia de "lógica de la investigación". Y aquí tenemos otro problema de confusión conceptual "contextos" y "lógicas" de descubrimiento y de verificación.

Los contextos de descubrimiento y verificación son momentos inevitables de una investigación en tanto etapas desde la concepción de Reichenbach (1934). Considerando que en el contexto de descubrimiento se alude a la forma en que los investigadores generan nuevas ideas, desde una perspectiva psicológica y social. Es decir, en el contexto de descubrimiento se desplegarían cómo surgen determinadas posiciones teóricas. Mientras que el contexto de justificación es la forma en que esas ideas se convierten en conocimiento válido a través de procesos metodológicos.

En la historia de la investigación y desde la perspectiva epistemológica se ha argumentado (Reinchebach, Popper, Hempel, Carnal, etc.) que sólo el contexto de justificación tiene un sustrato epistemológico lo "suficientemente sólido" para el desarrollo de una investigación.

Ahora bien, en la década de 1970 sociólogos de Edimburgo forman una organización llamada "Los amigos del descubrimiento" cuyo principal mentor será Thomas Nickles. Ese hito podría ser considerado como el 
traspaso del contexto a la lógica. Dado que a partir de este grupo se comienza a cuestionar la posición de la epistemología y de la historia de la ciencia predominante que sólo consideraban como legítimo la lógica de justificación o verificación. Y es aquí donde se produce la gran confusión epistemológica entre lógica y contexto. Los Amigos del Descubrimiento toman la categoría de verificación para cuestionarla, pero refiriéndose a ella como lógica.

En otras palabras, se reemplazó a-históricamente la categoría de lógica por la categoría histórica de contexto. Entendiendo, en términos de $\mathrm{H}$. Reinchebach, que siempre existirán contextos de descubrimiento y verificación, dado que son etapas por las que atraviesa inevitablemente el investigador (a) cuando se producen los descubrimientos en investigación y, (b) el modo en que se justifican. Esto se da porque en el proceso histórico, particularmente en el pasaje del monismo ontológico "del método científico" al pluralismo de "los métodos científicos" produjo, por acarreo, una confusión que viene particularmente de los "amigos del descubrimiento" y que tiene que ver con confundir los contextos con las lógicas. Como dijimos, los contextos son etapas del proceso personal del investigador, podríamos decir etapas psicológicas y las lógicas tienen que ver con el posicionamiento epistémico del investigador, esto es, en cuál de ellas se situará el énfasis: el descubrimiento o la verificación. En la decisión epistémica del investigador de optar por una u otra lógica surgirá la decisión de optar por uno u otro EE o viceversa. En este sentido Nickles plantea que durante ese período se comienza a dudar de "la lógica infalible de la prueba y la justificación en el sentido de un simple conjunto de reglas lógicas para la aceptación y rechazo de teorías” (1980:8).

Ahora bien, aceptando la presencia de las lógicas de descubrimiento y justificación, debemos considerar que se convierten en opciones en tanto decisiones epistemológicas (no nos referimos aún a la metodología). La cuestión es dónde, en qué LÓGICA el investigador decide instalar el énfasis y allí nos topamos nuevamente con una decisión epistemológica, considerando que estas lógicas pertenecen a caminos diversos para llevar a cabo una investigación. Consideremos a Samaja en relación a la pregunta y/o hipótesis y su vinculación con las lógicas de descubrimiento y verificación: "Las actividades de todo proceso de investigación pueden agruparse de acuerdo a dos criterios diferentes (1) según que las analicemos desde el punto de vista del descubrimiento (2) o desde el punto de vista de la validación o justificación" (1995:23). Y es aquí donde nos queremos detener brevemente.

En las lógicas descubrimiento y verificación se ponen en juego los "pares lógicos" o "modos suposicionales" (Goetz y Le Compte 1988). Los pares lógicos de la lógica de verificación serían: deducción - verificación explicación. En tanto los pares lógicos de la lógica de descubrimiento serían: inducción - generación de teoría-comprensión.

Es en esta decisión epistemológica, esto es, el énfasis en la lógica de justificación y verificación o de descubrimiento, donde se teje el EE del objeto de estudio.

\section{Lógicas de verificación y descubrimiento: pregunta o hipótesis}

Es así que podemos vincular lógicas de descubrimiento y verificación a los modos suposicionales y consecuentemente a los modos de construcción de una pregunta y una hipótesis como EE del objeto de estudio. Entendiendo que las dos lógicas son irreductibles entre sí. Los intentos de complementar una lógica a otra ignoran los modos posibles de construcción del conocimiento en una investigación, particularmente porque son dos maneras de conocer que tienen principios funcionales y componentes de análisis propios. 
Veamos algunos componentes de un proyecto según las lógicas.

\section{La función de la teoría}

a) lógica de verificación

Está más cerca de ser hipotético deductiva y de buscar explicación, verificación de teoría y generalización estadística. En esta lógica es importante comenzar con un sistema teórico, identificando variables que se definen teórica y operacionalmente. La definición operacional consiste en enunciar los procedimientos (instrumentos) con los cuales obtendrá determinada información empírica (indicadores). Según Goetz y Le Compte, "en cierto sentido, los investigadores deductivos pretenden encontrar datos que corroboren una teoría" (1988:30).

b) lógica de descubrimiento

Está más cerca de enfatizar la inducción analítica y de buscar la generación de teoría y la especificidad. La teoría orienta el trabajo en terreno con el propósito de generar teoría a partir del mundo empírico. Se busca y se trabaja con la teoría para: descubrir categorías (clases), sus propiedades y las relaciones entre clases en la construcción de una trama diferente, que trace a una unidad de sentido diferente. Ir relacionando con las teorías existentes a lo largo del proceso en terreno. Esta lógica se apoya en la "sensibilidad teórica" (Glazer y Strauss). La sensibilidad teórica es la habilidad de reconocer qué es importante en los datos y darle su significado. Esto permite la pregunta y re-pregunta continua. Según Goetz y Le Compte, "los investigadores inductivos intentan descubrir una teoría que explique sus datos" (1988:30).

\section{La deducción}

a) lógica de verificación

La deducción comienza con un sistema teórico, desarrolla hipótesis y definiciones operacionales de las proposiciones y conceptos de la teoría a través de un proceso de abstracción decreciente y los aplica empíricamente a algún conjunto de datos. En cierto sentido, los investigadores deductivos pretenden encontrar datos que verifiquen su teoría. Según Mardones, la deducción "consiste en deducir enunciados acerca de los fenómenos a partir de las premisas que incluyan o contengan a los principios explicativos" (1991:22).

b) lógica de descubrimiento

La deducción no es propia de la lógica de descubrimiento. Dado que los pasos que se requieren para realizar una investigación desde el razonamiento deductivo son de índole hipotéticos, es decir, se posee conocimiento previo y se infiere una conclusión que necesitará ser verificada. Y en la lógica de descubrimiento -como hemos señalado- no hay, metodológicamente, respuesta previa a ser verificada, es decir: no hay hipótesis.

\section{La inducción}

a) lógica de verificación 
La inducción no es propia de la lógica de verificación. Considerando que Carnap acuñó el término inducción como sinónimo de inferencia no-deductiva, esto es, una inferencia cuya conclusión no se sigue necesariamente de las premisas, por esta razón la inducción no cumple los requisitos de la lógica de verificación, que, como hemos mencionado, parte de una hipótesis que luego se verificará o no. Para esto se requiere del procedimiento deductivo, donde se parte de una premisa (hipótesis) para llegar a una conclusión, dado que la deducción es un encadenamiento formal, lógico y riguroso de proposiciones. Uno de los pasos claves de ese encadenamiento es la verificación, con la cual se obtendrá la conclusión. En este sentido el propio Popper (1965) postuló la imposibilidad de verificar una inducción.

b) lógica de descubrimiento

La investigación puramente inductiva, por el contrario a la deductiva, se inicia con conceptos generales y proposiciones amplias que orientan la focalización del objeto y de la pregunta. Se sumergen en el trabajo en terreno y se van construyendo en un movimiento en espiral: de lo empírico a la teoría y de la teoría a lo empírico. Se está interesado en desarrollar proposiciones: enunciados de los hechos inductivamente derivados a partir de un riguroso y sistemático análisis de datos. A través del examen de los casos semejantes y diferentes que han sido analizados se busca desarrollar un esquema conceptual, una teoría comprensiva. Como dice Mardones, la inducción "consiste en obtener principios explicativos a partir de los fenómenos que se han de explicar" (1991:22).

\section{La verificación y la explicación}

a) lógica de verificación

La búsqueda de la verificación consiste centralmente en comprobar que una hipótesis es válida o aplicable a varios conjuntos. El fin de la investigación verificativa o del énfasis puesto en la verificación, no sólo consiste en determinar si se cumple o no las relaciones entre variables prevista en una hipótesis, sino también el universo donde dicha proposición es aplicable con un mínimo margen de error. Como dicen Goetz y Le Compte, "el fin de la investigación verificativa no sólo consiste en determinar la medida en que se cumple una proposición, sino también el universo de poblaciones al que ésta es aplicable” (1988:30).

b) lógica de descubrimiento

La verificación y explicación no es propia de la lógica de descubrimiento.

\section{5. comprensión}

a) lógica de verificación

La comprensión no es propia de la lógica de verificación. Y aquí nuevamente una aclaración. No significa que con las hipótesis no se comprenda, pero estamos haciendo referencia a lógicas y categorías epistemológicas.

b) lógica de descubrimiento

El énfasis en el contexto de descubrimiento se centra en la identificación de categorías y proposiciones a partir de una base de información empírica. La obra clásica de Glaser y Strauss (1967), donde se desarrolla un modo de investigación centrado en la generación de teoría se llama justamente The discovery of grounded theory. Strategies for qualitative research. Como dicen Goetz y Le Compte: "Por su parte, la 
investigación generativa se centra en el descubrimiento de constructos y proposiciones a partir de una o más bases de datos o fuentes de evidencia" (1988:30).

Para comprender los cinco puntos señalados tomaremos el ejemplo anteriormente mencionado y obsérvese la hipótesis y la pregunta para 1) la función de la teoría, 2) la deducción, 3) la inducción, 4) la verificación y la explicación y, finalmente, 5) la comprensión.

Lógica de Verificación. Hipótesis

Ejemplo: Los alumnos de autorregulación alta tienen mejor desempeño académico que los alumnos de autorregulación baja.

Lógica de Descubrimiento. Pregunta.

Ejemplo: ¿Qué relación existe entre la autorregulación de los alumnos y el desempeño académico?

La pregunta claramente no responde a la lógica de verificación, particularmente por el modo en que se construye. Considerando que la pregunta por definición -a diferencia de la hipótesis- posee un alto grado de incertidumbre sobre lo que se desea conocer, genera la imposibilidad de verificar. Por esta razón es propia de la lógica de descubrimiento, dado que permite establecer cierta flexibilidad para explorar el fenómeno. Por otro lado, la hipótesis, respondiendo a la lógica de verificación, supone enunciados de asociación o relación entre variables (independientes, intervinientes, y dependientes). Se plantea la hipótesis en términos de verificación.

Ahora bien, debemos considerar que existe cierta ligazón, en lo que denominamos metodologías y lógicas, pero nunca de modo causal o inherente. Aunque no nos detendremos demasiado en esta cuestión, es necesario plantear que no existen metodologías mejores o peores que otras (Bechhofer 1996) o como afirma Scribano (2000) estas no son más que estrategias instrumentales, considerando que muchas polémicas se resolverían si aceptase que existe mala y buena investigación con ambas estrategias metodológicas.

Esto es, la metodología se desprende de determinado modo de construcción del objeto de estudio. Una metodología cuantitativa tendrá mayor relación con el modelo de verificación y un objeto de estudio construido tomando como eje la hipótesis. Ruth Sautu (Wainerman y Sautu 2001) explica: “La metodología cuantitativa es la adecuada cuando el propósito es describir la distribución de rasgos, opiniones, conductas, etc. o poner a prueba una hipótesis" (p.236) (la negrita es mía). Siguiendo con Sautu, señala que "en la investigación cuantitativa la teoría sustantiva referida al tema de estudio está presente desde el inicio como sistema clasificatorio de conceptos, regularidades empíricas, modelos causales que postulan relaciones entre variables" (p.234). En cambio refiriéndose a la lógica cualitativa de una investigación la autora señala: "que son apropiadas cuando el investigador se propone investigar la construcción social de significados, las perspectivas de los actores sociales, las condiciones de la vida cotidiana o describir la realidad" (p.236) (la negrita es mía). Y así explica que la cualitativa "parte de un conjunto menos específico de conceptos... y los elabora y reelabora en el curso de la investigación" (p.234).

Finalmente decimos, siguiendo con el hilo argumental de este artículo y teniendo en cuenta cómo se construye una hipótesis y cómo una pregunta como EE de la investigación y como componente del objeto de estudio, y, teniendo en cuenta también ambas lógicas, de verificación y descubrimiento: 
1) Una pregunta no se verifica. Se responde.

2) Una hipótesis se contrasta, se afirma o niega. En fin, se verifica o justifica. A tal punto que Popper (1965) denomina este proceso como corroboración (3).

Esto es clave, y es aquí donde muchos epistemólogos generan confusión temática. Obsérvese esta frase del prestigioso epistemólogo Gregorio Klimovsky: “La hipótesis al ser formulada se halla en estado de pregunta, y por lo tanto, para dejar de serlo deberá pasar por la verificación o refutación. Por lo tanto, la hipótesis se convierte en el corazón de la metodología de la concepción hipotética de la ciencia; puesto que frente a una pregunta se derivan una o más hipótesis" (2001:17).

En primer lugar es necesario destacar lo que venimos diciendo, pero en esta ocasión en términos de Klimovsky: la hipótesis se convierte en el corazón de la metodología de la concepción hipotética de la ciencia. En segundo lugar, aquí tenemos otra dificultad, cuando se confunde en términos metodológicos la pregunta con la hipótesis, y aquí es la categoría de pregunta que se utiliza desde el sentido común. La hipótesis no se construye del mismo modo que una pregunta. En este sentido, una pregunta o una hipótesis son el EE del objeto de investigación, pero es un error metodológico afirmar que de la "pregunta" se desprende la hipótesis.

Así podemos continuar con el análisis que desarrolla Samaja cuando explica que "el examen y discusión de las hipótesis que evocan las preguntas examen de las corazonadas" (2005:27). Sin duda que "la corazonada" de Samaja no es strictu sensu la hipótesis en el sentido metodológico.

\section{Conclusiones}

Hemos tratado de argumentar desde una perspectiva epistemológica la imposibilidad de conjugar en un mismo objeto de investigación dos ejes que pertenecen a lógicas diferentes: la hipótesis y la pregunta.

Consideramos que esta situación no ha sido advertida y analizada con detenimiento. En este sentido Pajares afirma: "La decisión acerca del uso de preguntas o hipótesis depende de factores tales como el propósito del estudio, la metodología utilizada y la audiencia a la cual se dirige la propuesta" (2007:3).

Esta cita muestra en primer término el uso de uno u otro eje. Sin embargo, entre los factores no se menciona la decisión epistemológica del investigador acerca de la decisión de optar por la lógica de verificación o de descubrimiento. Y aquí si es necesario volver a la cuestión de las metodologías cualitativas y cuantitativas, considerando, aunque no de modo excluyente, un investigador que utilice hipótesis y se sitúe con mayor énfasis en la lógica de verificación, quien se sentirá más cómodo trabajando con la metodología cuantitativa, por las particularidades de la construcción de hipótesis y la lógica de verificación. Y es allí donde la metodología no es solo una metodología, sino epistemetodología, dado que la metodología no es un componente aislado de las decisiones epistemológicas, sino que conforma una red de componentes que constituyen el proyecto de investigación. La metodología se convierte en una epistemetodología en la medida que está enhebrada a los otros componentes de la investigación. Por sí sola y, por ejemplo a los efectos de la enseñanza, son sólo técnicas metodológicas. La epistemetodología la defino como la decisión epistemológica en relación al EE y la lógica de descubrimiento o verificación y la posterior decisión de la inclusión de determinadas metodologías. 
Como hemos dicho, su inclusión no es mecánica ni causal: énfasis en la lógica de verificación = metodología cuantitativa o énfasis en la lógica de descubrimiento = metodología cualitativa. Pero la metodología forma parte de la posición epistémica del investigador en la medida que se vincula con otros componentes.

Es necesario dejar claro que la posición epistemológica (positivista, crítica, post-estructuralista, entre otros) no se cristaliza en la opción por la pregunta o hipótesis como eje de la investigación y parte de la construcción del objeto de investigación. Tal como afirma Reguillo, "en una investigación pueden utilizarse métodos de índole cualitativa y situarse en un enfoque absolutamente positivista" (1999:23).

Aunque no excluimos el formato de hipótesis como eje de la investigación en ciencias sociales es necesario considerar que en las últimas décadas el objeto de estudio de las ciencias sociales está desplegando un proceso transformación singular. Siguiendo el influjo de llya Prigogine, Pablo González-Casanova señala que la "complejidad obliga a cambiar los comportamientos epistemológicos" (2004:124) de la investigación de las ciencias sociales. Ya no se trata de la búsqueda de certidumbres, de leyes determinantes, ahora la ciencia define el proceso de investigación como "una acción en busca de posibilidades" creativas. En otro contexto, Niklas Luhmann a inicios de la década del sesenta ya planteaba una idea similar señalando que el fin del conocimiento social es "hacer comprensible la acción como posibilidad", y no el establecimiento de la acción según regularidades inalterables y deterministas (1973:40).

Varios colegas epistemólogos e investigadores, al momento de intercambiar las ideas que se sostuvieron en este artículo, me plantearon "pero yo no uso hipótesis en ese sentido, no me ubico en la lógica de verificación" a lo que yo respondía "si no utiliza hipótesis en ese sentido, pues utilice pregunta. Llámelo de otro modo". En un momento al comenzar a escribir este artículo había pensado en el siguiente título: "Ilamando las cosas por sus nombre: acerca de los componentes de un proyecto de investigación" dado que lo interesante de esto es que se encuentran en muchas investigaciones preguntas mal formuladas, es decir, preguntas formuladas como hipótesis. $\mathrm{Y}$ es allí donde se comienza a observar la incongruencia metodológica. $\mathrm{O}$ en otros casos, el uso de hipótesis pero utilizada con la lógica de la pregunta. Una u otra situación contribuye a la falta de consistencia de un proyecto de investigación.

\section{Notas}

(1) Conociendo las diferencias que diversos autores (Nuñez Flores 2008, Tamayo 1994, Sabino 1996, Hernández Sampieri y otros 2003) han realizado sobre ambos término. Sin embargo a los efectos de este artículo se utilizan como sinónimos.

(2) En nuestro relevamiento hemos visto estructuras curriculares similares en Argentina, Uruguay, Colombia, México y Brasil. Se desarrollan en las carreras de ciencias sociales al menos dos seminarios - cursos materias de metodología, con diversos nombres. Pero existe un nivel I y un nivel II. En los casos de carrera de grado (pregrado) donde se debe entregar una Tesina para la obtención del título de Licenciado, se desarrolla en general un Taller de Tesina o Taller de elaboración de trabajo final. Y en el caso de las maestrías sucede lo mismo, existe un primer espacio curricular como metodología o epistemología de las ciencias sociales y a posteriori un Taller de preparación-elaboración del Proyecto de Tesis. Por lo tanto podemos afirmar que un alumno posgraduado tuvo cinco materias metodológicas-epistemológicas. Por esta razón consideramos importante la advertencia que hicieran Bourdieu, Chamboredon y Passeron (1994) cuando afirman que: "A la tentativa que siempre surge de transformar los preceptos del método en recetas de cocina científica o en objetos de laboratorio, sólo puede oponérsele un ejercicio constante de vigilancia 
epistemológica que, subordinando el uso de técnicas y conceptos a un examen sobre las condiciones y los límites de su validez, proscriba la comodidad de una aplicación automática de procedimientos probados y señale que toda operación, no importa cuán rutinaria y repetida sea, debe repensarse a sí misma y en función del caso particular" (p.16).

(3) La corroboración, en la epistemología de Popper, es el estado de una teoría luego de que se la ha sometido a prueba y no se ha logrado falsarla.

\section{Bibliografía}

Ander-Egg, E. 1995. Cómo elaborar un proyecto: guía para diseñar proyectos sociales y culturales. Buenos Aires: Lumen.

Andrade, L. 2007. Del tema al objeto de investigación en Hugo Zemelman. Cinta moebio 30: 262-282.

Armstrong, R.L. 1974. Hypotheses: Why? When? How? Phi Delta Kappan 54: 213-214.

Ayala, M. 2001. Tipos de razonamiento y su aplicación estratégica en el aula. México: Universidad Virtual. ITESM

Becerra, A. 1994. Problema, problemática. Un primer esbozo de la teoría de la problematización. Caracas: UPEL, IPC.

Bechhofer, F. 1996. Quantitative research in British sociology: has it changed since 1981? Sociology 30(3): 583-591.

Barriga, O. y Henríquez, G. 2003. La presentación del objeto de estudio. Cinta moebio 17: 1-20.

Blumer, H. 1954. What is wrong with social theory? American Sociological Review 19: 3-10.

Bolívar, A. 2002. ¿De nobis ipsis silemus?: Epistemología de la investigación biográfico-narrativa en educación. Revista Electrónica de Investigación Educativa 4(1): 1-26.

Borsotti, C. 2008. Temas de metodología de la investigación en ciencias sociales empíricas. Buenos Aires: Editorial Miño y Dávila.

Bowen, G. 2006. Grounded theory and sensitizing concepts. International Journal of Qualitative Methods $5(3): 12-23$.

Bourdieu, P., Chamboredon, J-C y Passeron, J-C. 1994. El oficio del sociólogo. Madrid: Siglo XXI.

Buendia, L; Colas, P. y Hernandez, F. 2000. Métodos de investigación en psicopedagogía. Madrid: McGrawHill.

Bunge, M. 1993. La ciencia, su método y su filosofía. Buenos Aires: Siglo XXI.

Glaser, B.G y Strauss, A.L. 1967. The discovery of grounded theory: Strategies for qualitative research. Chicago: Aldine Publishing Company. 
Goetz J.P y Le Compte, M.D. 1988. Etnografía y diseño cualitativo en investigación educativa. Madrid: Morata.

González-Casanova, P. 2004. Las nuevas ciencias y las humanidades. De la academia a la política. Barcelona: Anthropos.

Goode, W. y Hatt, P. 1970. Métodos de investigación social. México: Editorial Trillas.

Hernández Sampieri, R. et. al. 2003. Metodología de la investigación. México: McGraw-Hill.

Kerlinger, F. 1979. Behavioral research: A conceptual approach. New York: Holt, Rinehart \& Winston.

Klimovsky, G. 2001. Las desventuras del conocimiento científico. Buenos Aires: AZ Editora.

Krathwohl, D. 1988. How to prepare a research proposal: Guidelines for funding and dissertations in the social and behavioral sciences. Syracuse, NY: Syracuse University Press.

Luhmann, N. 1973. Ilustración sociológica y otros ensayos. Buenos Aires: Sur.

Mardones, J.M. 1991. Filosofía de las ciencias humanas y sociales. Barcelona: Editorial Anthropos.

McMillan, J. y Schumacher, S. 2005. Investigación educativa. Madrid, Pearson: Addison Wesley.

Mejía, E. (Comp.) 2005. Metodología de la investigación científica. Lima: Centro de producción imprenta de la UNMSM.

Méndez, C. 1994. Metodología: Guía para la elaboración de diseños de investigación en ciencias económicas, contables y administrativas. Bogotá: McGraw-Hill.

Nickles, T. (ed.). 1980. Scientific discovery, logic and rationality. Boston: Reidel.

Nuñez Flores, M. 2008. Estrategia y técnica del diseño de investigación. Revista Investigación Educativa 12(21): 33- 41 .

Orozco, G. 2005. Construcción del objeto de investigación. Revista de Educación y Desarrollo 7: 41-50.

Pajares, F. 2007. Los elementos de una propuesta de investigación. Evaluar 7: 47-60.

Popper, K.R. 1965. La lógica de la investigación científica. Madrid: Editorial Tecnos.

Reichenbach, H. 1934. Experience and prediction: an analysis for the foundations and the structure of knowledge. Chicago: University of Chicago Press.

Reguillo, R. 1999. De la pasión metodológica o de la (paradójica) posibilidad de la investigación. En: Mejía, A. y Sandoval, A. (eds.) Tras las venas de la investigación cualitativa. Perspectivas y acercamientos desde la práctica. Guadalajara: ITESO, pp. 17-38.

Sabino, C. 1996. El proceso de investigación. Buenos Aires: Lumen Humanitas.

Salkind, N. 1999. Métodos de investigación. México: Prentice-Hall Hispanoamericana. 
Samaja, J. 2005. Epistemología y metodología: Elementos para una teoría de la investigación científica. Buenos Aires: Eudeba.

Samaja, J. 1995. El proceso de la ciencia. Una breve introducción a la investigación científica. Buenos Aires: Secretaría de Investigación y Posgrado UBA.

Sampieri, R. et.al. 2003. Metodología de la investigación. México: Mc Graw-Hill.

Scribano, A. 2000. Reflexiones epistemológicas sobre la investigación cualitativa en disciplinas sociales. Cinta moebio 8: 1-20.

Sirvent, M. 1999. Problemática actual de la investigación educativa. Revista del Instituto de Investigaciones en Ciencias de la Educación 8(14): 64-75.

Tamayo, M. 1994. El proceso de investigación científica. México: Limusa.

Wainerman, C. y Sautu, R. (comps.) 2001. Acerca de la formación de investigadores en ciencias sociales. Buenos Aires: Lumiere

Zemelman, H. 1987. Conocimiento y sujetos sociales. Contribución al estudio del presente. México: El Colegio de México.

Zemelman, H. 1992. Los horizontes de la razón I: Dialéctica y apropiación del presente. Barcelona: Anthropos/El Colegio de México.

Recibido el 25 May 2011

Aceptado el 5 Sep 2011 